\title{
The Perioperative Medicine Consult Handbook
}




\section{The Perioperative Medicine Consult Handbook}

\section{Second Edition}

\section{Molly Blackley Jackson, M.D.}

Division of General Internal Medicine, Department of Medicine, University of Washington Medical Center

Seattle, WA, USA

\section{Somnath Mookherjee, M.D.}

Division of General Internal Medicine, Department of Medicine, University of Washington Medical Center

Seattle, WA, USA

\section{Nason P. Hamlin, M.D., F.A.C.P.}

Division of General Internal Medicine, Department of Medicine, University of Washington Medical Center

Seattle, WA, USA

\section{Springer}


Molly Blackley Jackson, M.D.

Division of General Internal Medicine

Department of Medicine

University of Washington Medical Center

Seattle, WA, USA
Somnath Mookherjee, M.D.

Division of General Internal

Medicine

Department of Medicine

University of Washington

Medical Center

Seattle, WA, USA

Nason P. Hamlin, M.D., F.A.C.P.

Division of General Internal

Medicine

Department of Medicine

University of Washington

Medical Center

Seattle, WA, USA

ISBN 978-3-319-09365-9

DOI 10.1007/978-3-319-09366-6

ISBN 978-3-319-09366-6 (eBook)

Springer Cham Heidelberg New York Dordrecht London

Library of Congress Control Number: 2014951510

(c) Springer International Publishing Switzerland 2013, 2015

This work is subject to copyright. All rights are reserved by the Publisher, whether the whole or part of the material is concerned, specifically the rights of translation, reprinting, reuse of illustrations, recitation, broadcasting, reproduction on microfilms or in any other physical way, and transmission or information storage and retrieval, electronic adaptation, computer software, or by similar or dissimilar methodology now known or hereafter developed. Exempted from this legal reservation are brief excerpts in connection with reviews or scholarly analysis or material supplied specifically for the purpose of being entered and executed on a computer system, for exclusive use by the purchaser of the work. Duplication of this publication or parts thereof is permitted only under the provisions of the Copyright Law of the Publisher's location, in its current version, and permission for use must always be obtained from Springer. Permissions for use may be obtained through RightsLink at the Copyright Clearance Center. Violations are liable to prosecution under the respective Copyright Law.

The use of general descriptive names, registered names, trademarks, service marks, etc. in this publication does not imply, even in the absence of a specific statement, that such names are exempt from the relevant protective laws and regulations and therefore free for general use.

While the advice and information in this book are believed to be true and accurate at the date of publication, neither the authors nor the editors nor the publisher can accept any legal responsibility for any errors or omissions that may be made. The publisher makes no warranty, express or implied, with respect to the material contained herein.

Printed on acid-free paper

Springer is part of Springer Science+Business Media (www.springer.com) 


\section{Dedication}

We dedicate this handbook to Chris Wong, for his foundational work on all previous editions.

We also thank the residents and students at the University of Washington, who inspire us to be lifelong teachers and learners. 


\section{Preface}

The goal of this handbook is to support our colleagues (medical providers, trainees, and students) in providing thoughtful, evidencebased perioperative patient care. In the pages that follow, we aim to provide a focused review of medical issues that arise around the time of surgery along with suggested management strategies based on both the science and art of perioperative medicine.

The foundation of excellence in the care of surgical patients is teamwork. Collaboration and clear communication between the perioperative medicine consultant, surgeon, anesthesiologist, primary and specialty providers, inpatient team (including nurses, pharmacists, therapists, etc.), and the patient improves care, advances the knowledge of each team member, and is incredibly enjoyable. This text was created in partnership with dozens of colleagues at the University of Washington Medical Center, Harborview Medical Center, and the Seattle Veterans Affairs Puget Sound Health Care System. It has been our great privilege to learn and serve our patients together.

Seattle, WA, USA

Molly Blackley Jackson Somnath Mookherjee

Nason P. Hamlin 


\section{Acknowledgments}

\section{Amit D. Bhrany, M.D.}

Assistant Professor

Department of Otolaryngology

University of Washington

Surgical Procedures Overview

\section{Flavia B. Consens, M.D.}

Associate Professor

Department of Neurology

University of Washington

Department of Anesthesiology and Pain Medicine

University of Washington

Obstructive Sleep Apnea and Obesity Hypoventilation Syndrome

\section{Anthony DeSantis, M.D.}

Clinical Associate Professor

Division of Metabolism, Endocrinology and Nutrition

Department of Medicine

University of Washington

Diabetes

\section{Cora Espina, M.N., A.R.N.P., C.W.C.N.}

Teaching Assistant

Division of Metabolism, Endocrinology and Nutrition

Department of Medicine

University of Washington

Diabetes

Michael F. Fialkow, M.D., M.P.H.

Associate Professor

Department of Obstetrics and Gynecology 
University of Washington

Surgical Procedures Overview

Beatrice Franco, R.N., B.S.N., O.C.N.

Clinical Nurse Coordinator

Seattle Cancer Care Alliance

Sickle Cell Disease

\section{Greg Gardner, M.D.}

Professor

Division of Rheumatology

Department of Medicine

Adjunct Professor

Department of Orthopaedics and Sports Medicine

Adjunct Professor

Department of Rehabilitation Medicine

University of Washington

Rheumatoid Arthritis, Systemic Lupus Erythematosus, Gout and Pseudogout

\section{Steven Goldberg, M.D.}

Clinical Associate Professor

Division of Cardiology

Department of Medicine

University of Washington

Ischemic Heart Disease

John L. Gore, M.D., M.S.

Assistant Professor

Department of Urology

University of Washington

Surgical Procedures Overview

Wei Hao, M.D., Ph.D.

Clinical Associate Professor

Division of Metabolism, Endocrinology and Nutrition

Department of Medicine

University of Washington

Diabetes

Irl B. Hirsch, M.D.

Professor

Division of Metabolism, Endocrinology and Nutrition

Department of Medicine

University of Washington

Diabetes 


\section{Reena Julka, M.D.}

Attending Physician

Central Dupage Hospital

Venous Thromboembolic Disease, Chronic Kidney Disease, Patients with Solid Organ Transplant

Janet Kelly, Pharm.D.

Clinical Professor

Department of Pharmacy

University of Washington

Diabetes

\section{Saurabh Khandelwal, M.D.}

Assistant Professor

Division of General Surgery

Department of Surgery

University of Washington School of Medicine

Bariatric Surgery, Surgical Procedures Overview

\section{Barbara A. Konkle, M.D.}

Professor

Division of Hematology

Department of Medicine

University of Washington

Disorders of Hemostasis

\section{Seth Leopold, M.D.}

Professor

Department of Orthopaedics and Sports Medicine

University of Washington

Surgical Procedures Overview

Daniel W. Lin, M.D.

Professor

Department of Urology

University of Washington

Surgical Procedures Overview

\section{Iris W. Liou, M.D.}

Assistant Professor

Division of Gastroenterology

Department of Medicine

University of Washington

Liver Disease 


\section{Frederick A. Matsen, M.D.}

Professor

Department of Orthopaedics and Sports Medicine

University of Washington

Surgical Procedures Overview

Suzanne B. Murray, M.D.

Associate Professor

Department of Psychiatry and Behavioral Sciences

University of Washington

Decision-Making Capacity

\section{Ashok Reddy, M.D.}

Robert Wood Johnson Foundation Clinical Scholar

Perelman School of Medicine at University of Pennsylvania

Valvular Heart Disease, Chronic Kidney Disease

\section{Cindy Sayre, Ph.D.(c), A.R.N.P.}

Associate Administrator, Patient Care Services \& Professional Practice University of Washington Medical Center

Diabetes

\section{Laurie A. Soine, Ph.D., A.R.N.P.}

Teaching Associate

Division of Cardiology

Department of Medicine

Teaching Associate

Division of Nuclear Medicine and Cardiology

Department of Radiology

University of Washington

Cardiovascular Risk Stratification

\section{Lauge Sokol-Hessner, M.D.}

Acting Instructor

University of Washington Medical Center

Seattle, WA, USA

(at publication)

Instructor

Harvard University Medical School

Cambridge, MA, USA

Postoperative Ileus 


\section{Matthew Sweet, M.D.}

Assistant Professor

Division of Vascular Surgery

Department of Surgery

University of Washington

Surgical Procedures Overview

\section{Renata R. Urban, M.D.}

Assistant Professor

Department of Obstetrics and Gynecology

University of Washington

Surgical Procedures Overview

\section{Gail A. Van Norman, M.D.}

Professor

Department of Anesthesiology and Pain Medicine

University of Washington

Decision-Making Capacity

\section{Christopher J. Wong, M.D.}

Assistant Professor

Division of General Internal Medicine

Department of Medicine

University of Washington

Beta-Blockers, Atrial Fibrillation, Cerebrovascular Disease, Epilepsy and Seizure Disorders, Venous Thromboembolic Disease, Postoperative Evaluation, Postoperative Fever 


\section{Contents}

Preface

vii

Acknowledgments ........................................................... ix

Contributors ……......................................................... xxii

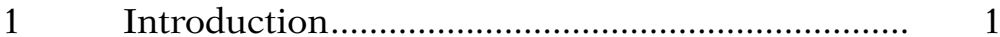

- General Guidelines............................................. 1

- Communication Is Vital!..................................... 2

- Documentation in the Medical Record Is Essential!

- "Comanagement" Versus "Consultation".......... 5

2 Styles of Medical Consultation ................................. 7

3 The Preoperative Evaluation ................................... 9

- Background ........................................................ 9

- Evaluation ....................................................... 9

- Assessment ....................................................... 15

- References ........................................................ 16

$4 \quad$ Perioperative Medication Management ................ 17

- Background ......................................................... 17

- Preoperative Evaluation ..................................... 17

- Perioperative Management................................. 18

- Specific Medicine Considerations ...................... 21

- References ......................................................... 24 
5 Anesthesia Pearls

- Background ...................................................... 27

- Perioperative Management................................ 27

- Pearls to Consider ............................................... 28

- Medicine Consult Notes..................................... 29

- When to Consider Involving Subspecialty Anesthesiologist ....................................... $\quad 30$

- Anesthesiology Terminology........................... 31

- References ....................................................... 31

$6 \quad$ Cardiovascular Risk Stratification...................... 33

- Background ................................................. 33

- Preoperative Evaluation ................................ 33

- References .......................................................... 41

$7 \quad$ Ischemic Heart Disease...................................... 43

- Background ................................................. 43

- Preoperative Evaluation ................................. 43

- Perioperative Management........................... 45

- References ............................................................ 46

$8 \quad$ Perioperative Beta-Blockers............................... 49

- Background ..................................................... 49

- Preoperative Evaluation ................................ 50

- Perioperative Management............................ 51

- References ................................................. 51

$9 \quad$ Atrial Fibrillation............................................. 53

- Background .............................................. 53

- Preoperative Evaluation ................................ 53

- Perioperative Management.............................. 54

- References ........................................................ 60

$10 \quad$ Hypertension ........................................... 63

- Background .............................................. 63

- Preoperative Evaluation ................................ 63

- Perioperative Management............................ 63

- References ........................................................... 66

11 Valvular Heart Disease ..................................... 67

- Background ................................................. 67

- Aortic Stenosis .................................................... 67 
- Mitral Stenosis ................................................ 69

- Aortic Regurgitation ....................................... 70

- Mitral Regurgitation ....................................... $\quad 70$

- Prosthetic Heart Valves..................................... 71

- References ...................................................... 73

12 Implantable Cardiac Electronic Devices............. 75

- Background .............................................. 75

- Preoperative Evaluation .................................. $\quad 77$

- Intraoperative Management........................... 79

- Postoperative Management ........................... 80

- References ..................................................... 81

13 Diabetes Mellitus ................................................. 83

- Background .................................................. 83

- Preoperative Evaluation ................................ 83

- Perioperative Management............................. 84

- References ...................................................... 90

$14 \quad$ Stress-Dose Steroids........................................ 93

- Background .............................................. 93

- Preoperative Evaluation .................................... 93

- Perioperative Management.............................. 94

- References ....................................................... 96

15 Thyroid Disease ................................................... 97

- Background ................................................ 97

- Preoperative Evaluation ................................. 97

- Perioperative Management............................. 98

- References ......................................................... 105

16 Liver Disease and Perioperative Risk .................. 107

- Background ................................................ 107

- Preoperative Evaluation ................................ 108

- Perioperative Management............................. 109

- References .................................................. 113

17 Inflammatory Bowel Disease ........................... 115

- Background ................................................ 115

- Preoperative Evaluation ................................... 115

- Perioperative Management............................ 116

- References ...................................................... 118 
18 Chronic Anticoagulation ..................................... 119

- Background ...................................................... 119

- Preoperative Evaluation ................................ 119

- Perioperative Management............................ 120

- References .................................................... 125

19 Disorders of Hemostasis .................................... 127

- Background ................................................ 127

- Preoperative Evaluation ............................... 127

- Perioperative Management............................ 129

- References ....................................................... 134

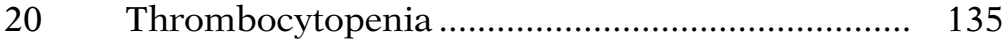

- Background ................................................ 135

- Perioperative Management............................. 135

- References ....................................................... 138

$21 \quad$ Heparin-Induced Thrombocytopenia................... 139

- Background ............................................... 139

- Perioperative Management............................. 139

- References ....................................................... 142

$22 \quad$ Anemia ....................................................... 143

- Background ..................................................... 143

- Preoperative Evaluation ................................. 143

- Perioperative Management................................ 144

- References ....................................................... 148

$23 \quad$ Sickle Cell Disease ................................................ 151

- Background ...................................................... 151

- Preoperative Evaluation ................................ 151

- Perioperative Management........................... 152

24 Cerebrovascular Disease .................................... 155

- Background ..................................................... 155

- Preoperative Evaluation ................................ 155

- Perioperative Management............................. 156

- References ......................................................... 157

25 Epilepsy and Seizure Disorders........................ 159

- Background .................................................. 159

- Preoperative Evaluation ................................... 159 
- Perioperative Management............................ 160

- References .................................................... 162

26 Parkinson's Disease ........................................... 163

- Background ...................................................... 163

- Preoperative Evaluation ................................. 163

- Perioperative Management............................. 164

- References ........................................................ 166

27 Pulmonary Risk Assessment and Management............................................... 167

- Background .................................................. 167

- Preoperative Evaluation .................................. 167

- Perioperative Management............................. 169

- References .................................................... 170

$28 \quad$ Asthma and COPD ............................................. 171

- Background ................................................ 171

- Preoperative Evaluation .................................. 171

- Perioperative Management............................. 172

- References ...................................................... 173

29 Obstructive Sleep Apnea and Obesity Hypoventilation Syndrome ................................ 175

- Background ..................................................... 175

- Preoperative Evaluation ............................... 176

- Perioperative Management............................ 177

- References .................................................... 177

30 Pulmonary Hypertension ................................ 179

- Background ................................................. 179

- Preoperative Evaluation ................................... 179

- Perioperative Management............................. 181

- References ....................................................... 184

$31 \quad$ Venous Thromboembolic Disease ........................ 185

- Background ....................................................... 185

- Preoperative Evaluation .................................. 185

- Perioperative Management............................ 188

- References ........................................................ 196 
$32 \quad$ Restrictive Lung Disease .................................... 199

- Background ...................................................... 199

- Preoperative Evaluation ................................ 199

- Postoperative Management .............................. 201

- References ........................................................... 201

33 Chronic Kidney Disease .................................... 203

- Background ................................................. 203

- Preoperative Evaluation ................................... 203

- Perioperative Management............................. 204

- References ..................................................... 206

$34 \quad$ Acute Kidney Injury ...................................... 209

- Background ................................................ 209

- Preoperative Evaluation .................................. 209

- Perioperative Management............................ 210

- References ....................................................... 213

35 Rheumatoid Arthritis ........................................ 215

- Background ............................................... 215

- Preoperative Evaluation ............................... 215

- Perioperative Management............................ 217

- References .................................................... 220

36 Systemic Lupus Erythematosus ........................ 221

- Background ............................................... 221

- Preoperative Evaluation .................................. 221

- Perioperative Management............................. 222

- References ..................................................... 223

$37 \quad$ Gout and Pseudogout........................................ 225

- Background ............................................. 225

- Preoperative Evaluation ................................ 225

- Perioperative Management............................ 226

- References ................................................... 227

$38 \quad$ Bariatric Surgery .............................................. 229

- Background .................................................. 229

- Preoperative Evaluation ................................ 229

- Perioperative Management............................ 232

- References ........................................................... 236 
39 Decision-Making Capacity 239

- Background

- Risk Factors for Loss of Decision-Making Capacity

- Assessment and Management

- References

40 Perioperative Care of Elderly Patients 243

- Background 243

- Preoperative Evaluation 243

- Perioperative Management 248

- References 249

41 Nutrition 251

- Background 251

- Preoperative Evaluation 251

- Perioperative Management. 252

- References 256

42 Patients with a Solid Organ Transplant 257

- Background ............................................... 257

- Preoperative Evaluation .................................. 257

- Perioperative Management............................. 258

- References ....................................................... 260

43 Substance Abuse and Dependence..................... 261

- Background ....................................................... 261

- Preoperative Evaluation .................................. 261

- Perioperative Management.............................. 262

- References .......................................................... 264

$44 \quad$ The Postoperative Evaluation............................... 265

- Background .................................................... 265

- Post-anesthesia Care Unit Assessment ........... 265

- Daily Postoperative Evaluation ....................... 267

- New Postoperative Consults .......................... 267

$45 \quad$ Postoperative Fever ............................................ 269

- Background ............................................... 269

- Perioperative Management............................ 269

- References ......................................................... 272 
46 Postoperative Delirium

273

- Background .................................................... 273

- Preoperative Evaluation ............................... 273

- Perioperative Management............................ 274

- References ........................................................ 277

$47 \quad$ Postoperative Ileus ....................................... 279

- Background ............................................... 279

- Preoperative Evaluation ............................... 280

- Perioperative Management............................. 280

- References ....................................................... 284

$48 \quad$ Surgical Procedures Overview ........................... 287

- Background .................................................... 287

- Orthopedic Surgery........................................ 287

- General Surgery .......................................... 291

- Gynecology and Gynecology-Oncology Surgery ........................................................... 294

- Urologic Surgery/Procedures ........................ 296

- Vascular Surgery ............................................... 299

- Head and Neck Surgery ................................... 301

- Neurosurgery .............................................. 302

- Ophthalmologic Surgery ................................. 303

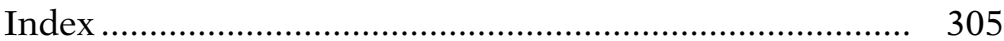




\section{Contributors}

Gabrielle Berger, M.D.

Division of General Internal Medicine, Department of Medicine, University of Washington, Seattle, WA, USA John H. Choe, M.D., M.P.H.

Division of General Internal Medicine, Department of Medicine, University of Washington, Seattle, WA, USA Paul B. Cornia, M.D.

Division of General Internal Medicine, Department of Medicine, University of Washington, Seattle, WA, USA

Sandra M. Demars, M.D.

Division of General Internal Medicine, Department of Medicine, University of Washington, Seattle, WA, USA

Joana Lima Ferreira, M.D.

Division of General Internal Medicine, Department of Medicine, University of Washington, Seattle, WA, USA

Lindsay Frank, R.D., C.N.S.D.

University of Washington, Seattle, WA, USA

Divya Gollapudi, M.D.

Division of General Internal Medicine, Department of Medicine, University of Washington, Seattle, WA, USA Anna L. Golob, M.D.

Division of General Internal Medicine, Department of Medicine, University of Washington, Seattle, WA, USA 
Nason P. Hamlin, M.D.

Division of General Internal Medicine, Department of Medicine, University of Washington, Seattle, WA, USA

Ronald Huang, M.D., M.P.H.

Division of General Internal Medicine, Department of Medicine, University of Washington, Seattle, WA, USA Molly Blackley Jackson, M.D.

Division of General Internal Medicine, Department of Medicine, University of Washington, Seattle, WA, USA

Kay M. Johnson, M.D., M.P.H.

Division of General Internal Medicine, Department of Medicine, University of Washington, Seattle, WA, USA

Elizabeth Kaplan, M.D.

Division of General Internal Medicine, Department of Medicine, University of Washington, Seattle, WA, USA Tyler Lee, M.D.

Division of General Internal Medicine, Department of Medicine, University of Washington, Seattle, WA, USA Jennifer R. Lyden, M.D.

Division of General Internal Medicine, Department of Medicine, University of Washington, Seattle, WA, USA

Karen A. McDonough, M.D.

Division of General Internal Medicine, Department of Medicine, University of Washington, Seattle, WA, USA

Kara J. Mitchell, M.D.

Division of General Internal Medicine, Department of Medicine, University of Washington, Seattle, WA, USA

Somnath Mookherjee, M.D.

Division of General Internal Medicine, Department of Medicine, University of Washington, Seattle, WA, USA

Brian S. Porter, M.D.

Division of General Internal Medicine, Department of Medicine, University of Washington, Seattle, WA, USA 
Ashok Reddy, M.D.

Robert Wood Johnson Foundation, Princeton, NJ, USA Perelman School of Medicine at University of Pennsylvani, Seattle, WA, USA

G. Alec Rooke, M.D.

Department of Anesthesiology and Pain Medicine, University of Washington, Seattle, WA, USA

Christina Ryan, M.D.

Division of General Internal Medicine, Department of Medicine, University of Washington, Seattle, WA, USA

Department of Neurological Surgery, University of Washington, Seattle, WA, USA

Nina Saxena, M.D.

Division of General Internal Medicine, Department of Medicine, University of Washington, Seattle, WA, USA

Sabeena Setia, M.D.

Division of General Internal Medicine, Department of Medicine, University of Washington, Seattle, WA, USA

Tara Spector, M.D.

Division of General Internal Medicine, Department of Medicine, University of Washington, Seattle, WA, USA

Rachel E. Thompson, M.D.

Division of General Internal Medicine, Department of Medicine, University of Washington, Seattle, WA, USA

Gail A. Van Norman, M.D.

Department of Anesthesiology and Pain Medicine, University of Washington, Seattle, WA, USA

Kelly Wentworth, M.D.

Division of Hospital Medicine, Department of Medicine, University of California San Francisco, San Francisco, CA, USA

Andrew A. White, M.D.

Division of General Internal Medicine, Department of Medicine, University of Washington, Seattle, WA, USA 
Christopher J. Wong, M.D.

Division of General Internal Medicine, Department of Medicine, University of Washington, Seattle, WA, USA

Jeanie C. Yoon, M.D.

Division of General Internal Medicine, Department of Medicine, University of Washington, Seattle, WA, USA 